\title{
Experimental Study of Propane-Fueled Pulsed Detonation Rocket
}

\author{
Frank K. Lu, ${ }^{*}$ Jason M. Meyers, ${ }^{\dagger}$ Donald R. Wilson ${ }^{\ddagger}$ \\ University of Texas at Arlington, Arlington, Texas 76019
}

\begin{abstract}
A major problem applying detonations into aero-propulsive devices is the transition of deflagration and weak detonation into $\mathbf{C J}$ detonation. The longer this transition, the longer the physical length of the engine must be to facilitate the propagation of the flame. However, lengthening of the detonation chamber can significantly increase weight, rendering the reduction of deflagration-to-detonation (DDT) and weak detonation transition length of great importance. One of the most common means of shortening these lengths is with the aid of a Shchelkin spiral. A simple helical apparatus, it was used in early single-shot detonation investigations to over-exaggerate wall roughness effects $^{1}$. It was through empirical investigations that the reduced DDT phenomenon was observed. The present investigation explored the possibility of applying such an apparatus into an intermittent pulsed detonation device using gaseous $\mathrm{C}_{3} \mathrm{H}_{8} / \mathrm{O}_{2}$. Results show significant improvements in comparison to cases without the spiral. Tests through a range of cycle frequencies up to $20 \mathrm{~Hz}$ in oxygen-propane mixtures at $1 \mathrm{~atm}$ demonstrated the feasibility of the Shchelkin spiral in a pulsed mode. The results also demonstrated the need to optimize the chamber's dimensions and various other associated processes to ensure that a detonation wave is sustained within the detonation chamber.
\end{abstract}

\section{Introduction}

$\mathrm{P}$ ulse detonation engines (PDEs) have recently been recognized as a promising propulsion technology that offers advantages in thermodynamic cycle efficiency, hardware simplicity, scability and reliability. ${ }^{1}$ PDEs use detonation combustion rather than deflagration and hence can yield rapid energy release and high specific power. In principle, PDE utilizes propagating detonation waves generated periodically within a duct, typically with secondary wave reflections, to produce large thrust.

An important technical challenge remains the ability to achieve consistent, repetitive detonations in a short distance. The direct initiation of detonation requires an inordinate amount of energy while a deflagration-todetonation transition (DDT) occurs at lower energies. For propulsion applications, the large energy requirement poses practical problems since such an energy source may not be readily available in a compact and lightweight package. Consideration therefore turns to an acceptable deflagration-to-detonation (DDT) length since it has been found that as long as transition occurs within the length of the detonation tube, the specific impulse obtained is the same as that from direct initiation. ${ }^{2}$ The

Trofessor, Mechanical and Aerospace Engineering Department, and Director, Aerodynamics Research Center, Box 19018, Associate Fellow AIAA.

*Research Associate, Aerodynamics Research Center; presently, Diploma Course Student, Von Karman Institute for Fluid Dynamics, Rhode-SaintGenèse, Belgium, Student Member AIAA.

†Professor, Department of Mechanical and Aerospace Engineering, Box 19018, Associate Fellow AIAA.

Copyright (C) 2003 by F.K. Lu, J.M. Meyers and D.R. Wilson. Published by the American Institute of Aeronautics and Astronautics, Inc. with permission. trade-off between energy and DDT length (or time) is crucial. A weak source would result in a long transition that causes problems associated with length, such as reduction in cycle frequency, and increased mixing time and engine weight. Most single-shot experiments at present yield transition lengths of $0.7-2 \mathrm{~m}$ that are considered to be too excessive to be practical.

Since it appears that some DDT must occur in practice, ways are sought to reduce the transition length. It is known that elevated pressure and temperature, reactants at stoichiometric conditions, high ignition energy and various enhancement devices such as Shchelkin spirals, orifice plates and a centerbody, reduce the DDT length. These techniques have been well studied in single-shot facilities. ${ }^{3-7}$ However, only a few reports exist on using these devices in a pulsed operation mode. ${ }^{8}$

As part of an ongoing program to develop pulse detonation applications, experiments were performed to investigate the behavior of a Shchelkin spiral in a pulsed detonation engine operating with propane and oxygen. A high-energy igniter is used in conjunction with the enhancement device. The study showed that a short DDT length was achieved, confirming single-shot observations. However, the existing fuel injection system could not fill the detonation chamber fast enough to sustain detonations at high frequencies. This revealed the need for a better understanding of the various gasdynamic processes in pulsed detonation in order to optimize these processes for high-frequency operation.

\section{Experiment Setup}

The experiments were performed in a high frequency pulse detonation rocket facility. This facility utilizes a mechanical rotary valve injection system for propane, oxygen and air, the first two being the propellants and 
the latter for purging the detonation chamber. The propellants were detonated using a high current, electric arc discharge. Near stoichiometric ratios were calibrated with the aid of two critical flow nozzles, one for propane and one for oxygen.

\section{Detonation Chamber}

The detonation engine is constructed of steel pipe with inside and outside diameters of $7.62 \mathrm{~cm}$ (3 in.) and 15.2 $\mathrm{cm}$ ( 6 in) respectively. Various lengths of 7.62, 15.2 and $30.5 \mathrm{~cm}$ (3, 6, and 12 in.) were available. Different lengths could be assembled through end flanges with rubber o-ring seals. Each segment had ports for proper instrumentation, such as pressure transducers, thermocouples, heat flux gauges or photo-detectors. The 30.48 $\mathrm{cm}$ (12 in.) sections allowed for four equally spaced instrumentation ports along the tube. Sections of $15.24 \mathrm{~cm}$ (6 in.) allowed for two ports, and $7.62 \mathrm{~cm}$ (3 in.) sections allowed for one port. Another $7.62 \mathrm{~cm}$ ( 3 in) section was used to support the mounting of the arc-plug igniter. Figure 1 is a schematic of the detonation chamber with a Shchelkin spiral installed. Sections were assembled to the injection end-plate to yield a total chamber length of $53.34 \mathrm{~cm}$ (21 in.). The first $7.62 \mathrm{~cm}$ (3 in.) section was used to mount the igniter $3.81 \mathrm{~cm}$ (1.5 in.) downstream of the injection wall. The following $30.48 \mathrm{~cm}$ (12 in.) and then $15.24 \mathrm{~cm}$ (6 in.) sections housed four ports and two ports, respectively, used for pressure transducer mounting. These are labeled $P_{2}-P_{7}$ in Fig. $1\left(P_{1}\right.$ is an

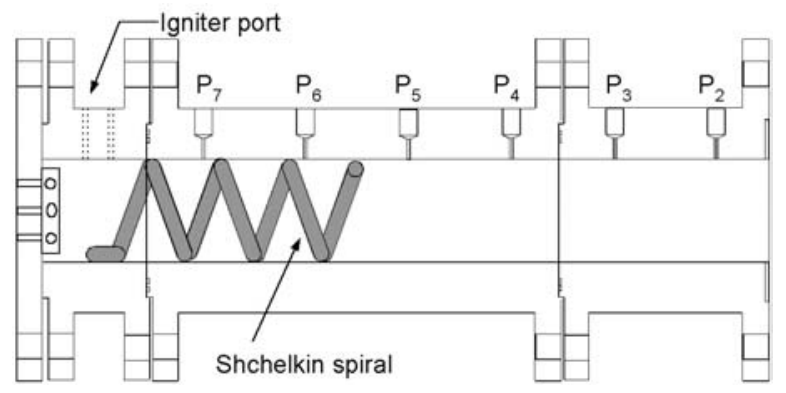

Figure 1. Detonation chamber with Shchelkin spiral installed.

atmospheric reference transducer.) The first instrumentation port $P_{7}$ is located $7.62 \mathrm{~cm}$ (3 in.) downstream of the igniter with each of the five successive transducer ports at $7.62 \mathrm{~cm}$ (3 in.) intervals. The Shchelkin spiral had a pitch of 15 degrees and wire diameter of $9.53 \mathrm{~mm}$ ( $3 / 8$ in). Blockage ratio, the area of the obstruction to the area of the clean cross-section, for the spiral was about 0.21 , relatively small when compared to other DDT experimental studies. ${ }^{6}$

\section{Ignition System}

A proper ignition system is one of the most crucial components for successful multi-cycle detonation experimentation. A high current, electrical discharge is a practical choice. An in-house ignition system, which can operate at up to $200 \mathrm{~Hz}$, was used in the experiments. It can produce a maximum of $28 \mathrm{~J} /$ pulse at low frequencies, which drops to $5 \mathrm{~J} /$ pulse at $200 \mathrm{~Hz}$. The pulse energy was estimated to be $13.5 \mathrm{~J}$ for the present experiments.

\section{Propellant Supply System}

The propane and oxygen sources were kept at a safe distance from each other and from the PDE. Flash arrestors were installed about $15 \mathrm{~m}$ upstream of the mass-flow meters to further ensure safety. The reactants were delivered to the detonation chamber via a mechanical rotary valve injection system. This valve was designed and fabricated in-house for fuel and oxygen injection, and for purging purposes. Gases were injected from the side opposite the drive gear and then distributed from three ports in a radial fashion from the internal rotating shaft. The three valves are mounted to the engine via a trapezoidal-shaped manifold (Fig. 2).

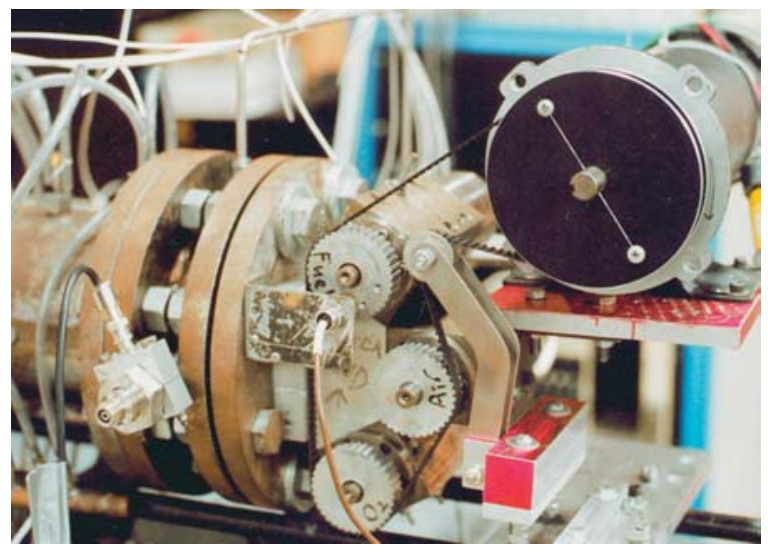

Figure 2. Rotary valve injection assembly.

The injection system directed the propellant and purge flows into the engine from the end wall. The gases were then forced into a swirling motion by an injection disk mounted on the end wall inside the detonation tube to enhance mixing. These injector ports can be seen schematically on the left end wall in Fig. 1. Due to the present drive gear radius and friction of the rotating system, the $0.5 \mathrm{hp}$ electric motor was only capable of driving the system up to a cycle frequency of $20 \mathrm{~Hz}$.

Determining the mass flow of the propellants in a pulsed flow was more involved than the partial pressure method used when charging up a single shot detonation experiment. Two Flow-Dyne Corp. (Fort Worth, Texas) critical flow nozzles were used for metering oxygen and propane flow rates. Steady, upstream values of pressure and temperature were used along with the area ratio of the convergent-divergent "critical flow" nozzle in the following relationship: 


$$
\dot{m}=K\left(A / A^{*}, P_{1}\right) \frac{P_{1}}{\sqrt{T_{1}}}
$$

for both propane and oxygen.

Calibration was carried out using flows of the actual propellant gases under cold flow (or "engine-off”) conditions. Choking of the nozzle is crucial for proper measurements. A steady pressure record by the upstream pressure transducers indicates that the nozzles were choked even as the injection system pulsed flow into the chamber. If oscillations occurred at the cycle frequency, then the flow nozzles were not choked. After the cold flow calibration was complete, mass flows were measured during hot flow (or "engine-on") conditions. When these mass flows were calculated and confirmed with that of the cold flow tests, the two pressure transducers and two thermocouples were removed to free memory for either higher resolution or longer duration data sampling.

Caution must be taken when using the measured mass flows because they were metered about $1.5 \mathrm{~m}$ upstream of the injection valves. Due to compressibility effects in the remaining tubing downstream of the flow nozzles, the calculated equivalence ratio of the injected species may not be exactly stoichiometric. Minor trimming of the regulator pressures from the estimated stoichiometric values were done until maximum engine performance was obtained.

\section{Test Conditions and Data Acquisition}

Experiments were conducted for configurations with the Shchelkin spiral at frequencies of 4.4, 6.9, 14.4 and 20 $\mathrm{Hz}$ using a stoichiometric propane/oxygen mixture at room conditions of $1 \mathrm{~atm}$ and $20^{\circ} \mathrm{C}$. The clean configuration was tested at $6.9 \mathrm{~Hz}$ only. Six PCB (Depew, New York) pressure transducers were used in the experiments. The data at 14.4 and $20 \mathrm{~Hz}$ operation were acquired by 12-bit digitizers at $100 \mathrm{kHz} / \mathrm{channel}$ (DSP Technology, Inc., presently part of Spectral Dynamics, San Jose, California). The data were sampled with a temporal resolution of $10 \mu \mathrm{s}$ resolution to fill the 512 ksample memory but this sampling rate seriously handicapped the ability to resolve pressure peaks. Experiments at 4.4 and $6.9 \mathrm{~Hz}$ used $1 \mathrm{MHz} /$ channel digitizers with a temporal resolution of $1 \mu \mathrm{s}$. In conjunction with a 2.048 Msample memory module, only $250 \mathrm{~ms}$ worth of data can be acquired. The large data files created were, unfortunately, not fully analyzed. The quandary encountered here is the need for high temporal resolution vis-à-vis limited storage and the data reduction effort.

The pressure transducers were water-cooled and recessed in the mounting ports to protect them. However, this procedure damped out the pressure peaks. Previous work has suggested that the wave speed, using a time-offlight (TOF) method, remains a good indicator of DDT, instead of relying on pressure peaks. ${ }^{9}$ The TOF method, when used with the $1 \mathrm{MHz}$ digitizers, yielded a resolution of $2 \mu \mathrm{s}$ for velocities of $2000 \mathrm{~m} / \mathrm{s}$, resulting in a 5 percent error. TOF data at 4.4 and $6.9 \mathrm{~Hz}$ were obtained

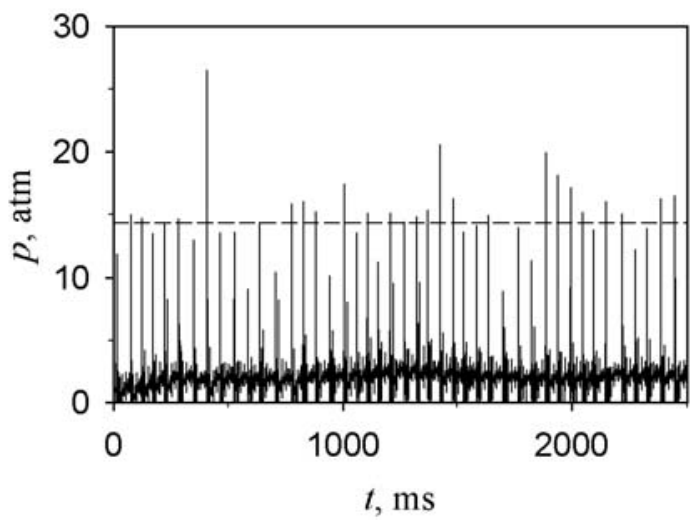

a. $2.5 \mathrm{~s}$ record (average pressure shown as dashed line)

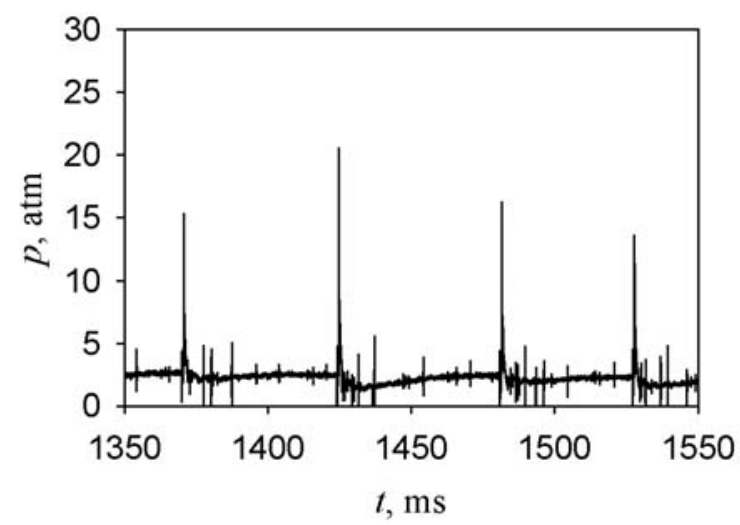

b. Enlargement showing a number of consecutive pulses

Figure 3. Pressure record of transducer 6 at $20 \mathrm{~Hz}$, with Shchelkin spiral.

with $1 \mathrm{MHz}$ digitizers, while those at 14.4 and $20 \mathrm{~Hz}$ were obtained with 100 digitizers.

\section{Results}

The ability of the test rig to obtain repetitive cycles is shown in Fig. 3a, which shows a $2.5 \mathrm{~s}$ window of data, obtained a few seconds after starting the test. Note that transducer No. 6 lies within the Shchelkin spiral. The data were acquired at a sampling frequency of 100 $\mathrm{kHz} /$ channel due to memory constraints. This was adequate for a cycle-to-cycle repeatability experiment of relative long sampling duration. Cycle-to-cycle repeatability shows significant overpressure levels of around $14.3 \mathrm{~atm}$ on average, albeit lower than the CJ level. (The CJ pressure obtained from the NASA CEA code is 35 atm for a stoichiometric oxygen/propane mixture at stan- 
dard conditions. ${ }^{10}$ ) The lower experimental value of peak pressure is partly due to the difficulty in resolving the peak, as mentioned above, and was also thought to be due to poor mixing. The latter difficulty will be discussed later. A narrow time window showing a number of consecutive pulses can be found in Fig. 3b. The enlargement shows that the characteristic shape of the pulses appears similar and comparable to those obtained by other investigators of single-shot detonation waves, namely, that the steep pressure rise is followed by a gentle expansion to sub-ambient.

Sample pressure profiles obtained at $6.9 \mathrm{~Hz}$ are shown in Fig. 4 for a clean configuration and one with a
Shchelkin spiral. The pressure profiles of the clean configuration (Fig. 4a) showed poor performance. The wave appeared to accelerate and transition to a detonation profile, as indicated by the $P_{6}$ record, but failed before reaching the open end. Amongst the numerous runs, not one achieved detonation within the detonation tube, with the wave velocity exceeding sonic but in general barely reaching 30 percent of the CJ level. A noticeable feature of the sub-CJ wave, as also observed in previous singleshot experiments, is a pre-compression before the sharp pressure front, for example, as seen in $P_{6}-P_{4}$ (Ref. 9). The sub-CJ pre-compression is also apparent in the $P_{7}$ record with the Shchelkin spiral installed (Fig. 4b).

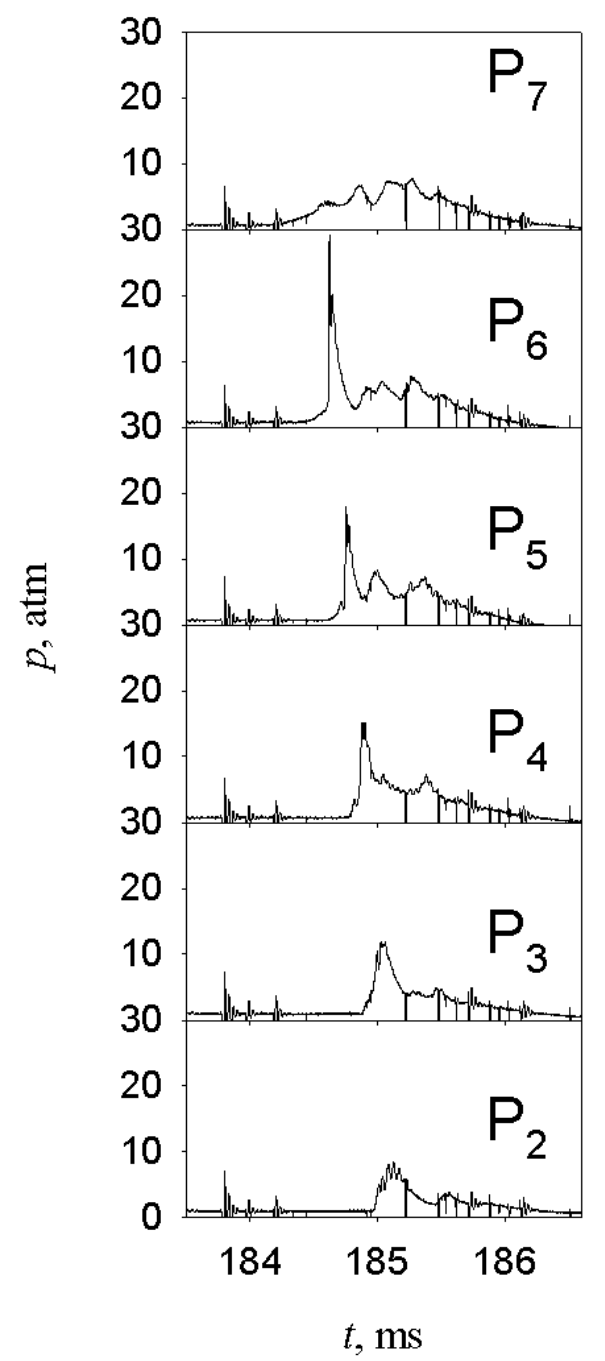

a. Clean configuration.

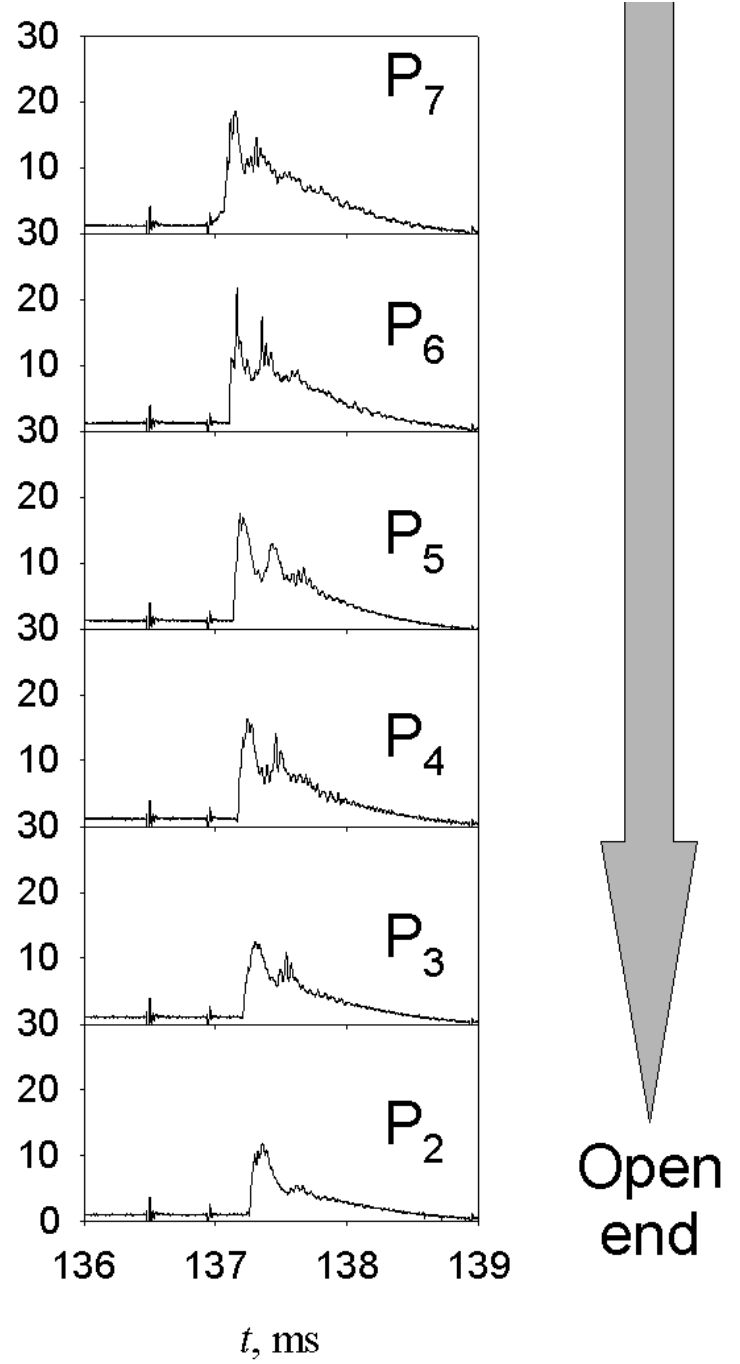

b. Shchelkin configuration.

Figure 4. Pressure profiles at $6.9 \mathrm{~Hz}$ cycle frequency. 
A reason for the poor performance may be poor mixing of the reactants, in addition to the usual suspicion of inadequate DDT length. At $6.9 \mathrm{~Hz}$, a window of about $40 \mathrm{~ms}$ (the time after the injection valve closed to the time of ignition) is available for mixing. The swirling disc used to enhance mixing may not be creating the desired level of turbulence to completely mix the reactants in such a short period.

In contrast, the wave evolution with the Shchelkin spiral showed more promise in reducing the DDT length. In the presented results, the profiles for $P_{6}-P_{4}$ showed a distinct pressure spike characteristic of a detonation wave. While the transducers were unable to obtain the peak pressure value, the wave propagation velocity appeared to be a good indicator of DDT. The propagation velocity for different waves for the two configurations is summarized in Fig. 5. The figure shows that the clean configuration achieves a subcritical detonation that ultimately reached about twice the sonic velocity at the tube exit whereas, with the Shchelkin spiral, the wave consistently reached the CJ velocity in a short distance near the end of the spiral. This velocity, however, was not sustained and the wave decelerated toward the open end of the tube. Moreover, Fig. 4 shows that the wave appeared to lose its characteristic detonative shape.

To understand why the Shchelkin spiral results did not show a sustained CJ velocity, the different cycle tests were compared. Samples of single pulses were chosen for comparison in Fig. 6. The figure shows a progressive decline in the ability to sustain a CJ velocity as the cycle frequency is increased. For example, at $4.4 \mathrm{~Hz}$, the wave accelerates and maintains itself whereas at $20 \mathrm{~Hz}$ the wave reached only a subcritical state. It is believed that the lower frequency allowed more time for injection and

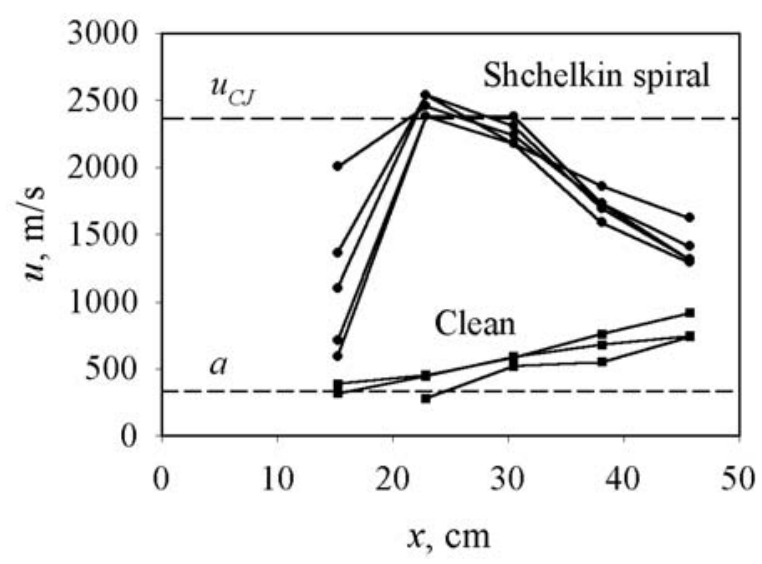

Figure 5. Detonation chamber with Shchelkin spiral installed, $6.9 \mathrm{~Hz}$ operation.

mixing to provide the better performance. Figure 7 also shows that the present configuration may actually be longer than necessary for developing a compact pulsed detonation device. Given proper injection and mixing,

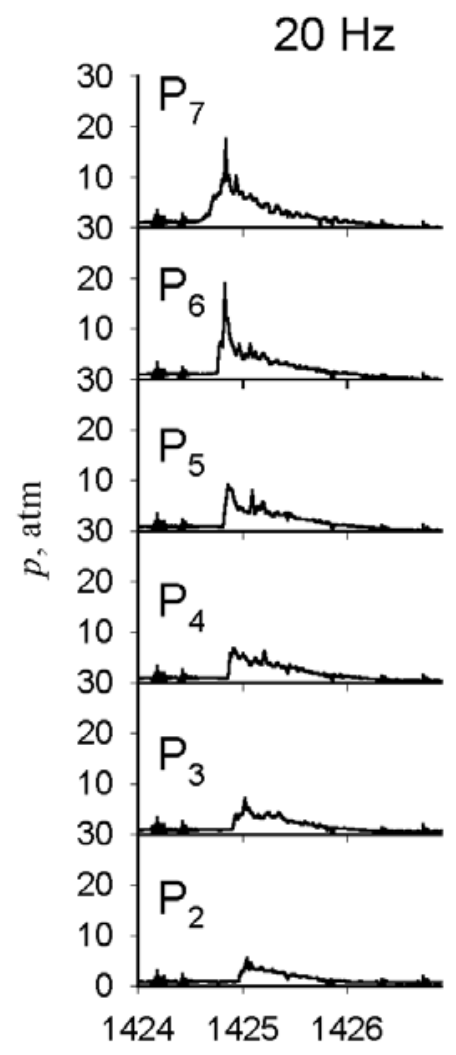

$14.4 \mathrm{~Hz}$

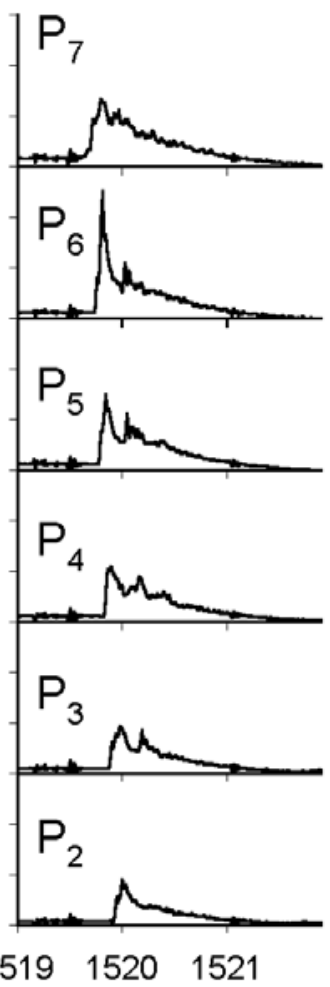

$6.9 \mathrm{~Hz}$

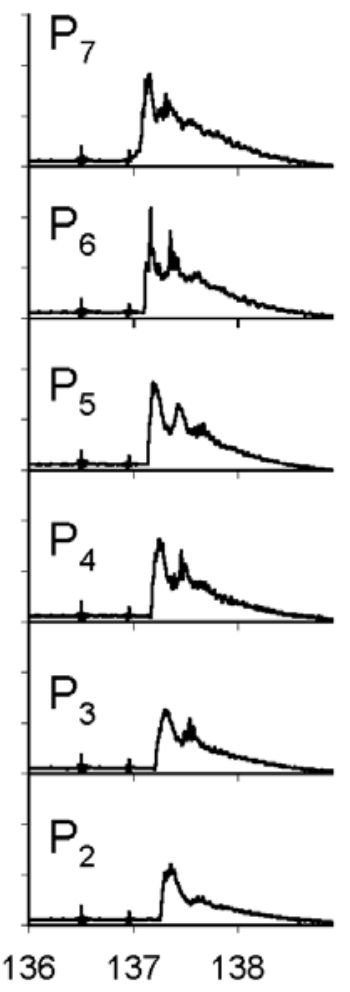

$4.4 \mathrm{~Hz}$

Closed

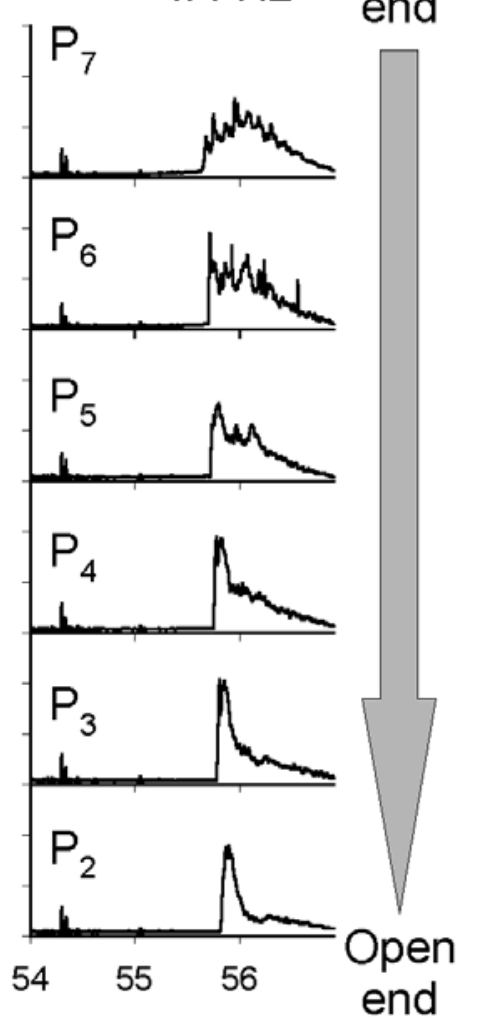

$t, \mathrm{~ms}$

b.

c. d.

Figure 6. Single-pulse propagation comparison at various frequencies for the Shchelkin spiral configuration. 
the Shchelkin spiral configuration yielded a CJ velocity in a length of about $20 \mathrm{~cm}$ (8 in.) from the closed end. Further experiments with closer transducer spacing are needed to provide a better DDT length measurement.

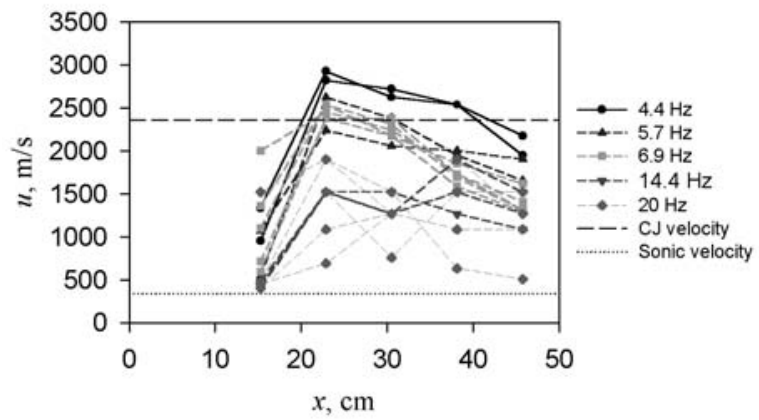

Figure 7. Wave propagation for Shchelkin spiral configuration at various frequencies.

\section{Conclusions}

Experiments were performed in a pulse detonation facility using a stoichiometric propane/oxygen mixture initially at ambient conditions. The experiments showed that detonations could be obtained in a short distance of 11-17 cm (4-7 in.) for modest cycle frequencies of 4-7 $\mathrm{Hz}$ with a Shchelkin spiral installed. At $14-20 \mathrm{~Hz}$, only strong intermittent overpressures are observed. Each pressure profile yielded weak pre-compressions followed closely with a relatively strong overpressure peak that quickly deteriorated after passing the Shchelkin spiral. The wave velocities, which provided a more reliable indicator of whether CJ conditions were achieved, also showed sub-CJ behavior at higher frequencies. This failure to attain CJ conditions was thought to be possibly due to improper filling and mixing of the detonation chamber from cycle to cycle since identical conditions at lower frequencies yielded strong evidence that CJ conditions were achieved.

\section{Acknowledgements}

Partial support of the research reported here came from the Texas Advanced Technology Program (Grant No. 14761051) and the U.S. Civilian Research and Development Foundation (Grant No. 26-7501-07).

\section{References}

\footnotetext{
${ }^{1}$ Kailasanath, K., "Recent Developments in the Research on Pulse Detonation Engines," AIAA Journal, Vol. 41, No. 2, 2003, pp. 145-159.

${ }^{2}$ Kiyanda, C.B., Tanguay, V., Higgins, A.J. and Lee, J.H.S., "Effect of Transient Gasdynamic Processes on the Impulse of Pulse Detonation Engines," Journal of Propulsion and Power, Vol. 18, No. 5, 2002, pp. 1124-1125.

${ }^{3}$ Knystautas, R. and Lee, J.H.S., "On the Effective Energy for Direct Initiation of Detonations," Combustion and Flame,
}

Vol. 27, 1976, pp. 221-228.

${ }^{4}$ Smirnov, N.N., Nikitin, V.F., Boichenko, A.P., Tyurnikov, M.V., and Baskakov, V.V., "Deflagration to Detonation Transition in Gases and its Applications in Pulsed Detonation Devices" in Gaseous and Heterogeneous Detonations: Science to Applications, ed. by N.N. Smirnov and G.D. Roy ENAS, Moscow, 1999, pp. 65-94.

${ }^{5}$ Schauer, F., Stutrud, J. and Bradley, R., "Detonation Initiation Studies and Performance Results for Pulsed Detonation Engine Applications," AIAA Paper 2001-1129, 2001.

${ }^{6}$ Lee, S.Y., Conrad, C., Watts, J., Woodward, R., Pal, S. and Santoro, R.J., "Deflagration to Detonation Transition Study Using Simultaneous Schlieren and OH PLIF Images," AIAA Paper 2000-3217, 2000.

${ }^{7}$ Lu, F.K. and Wilson, D.R., "., "Some Perspectives on Pulse Detonation Propulsion Systems," Paper No. 1051, $24^{\text {th }}$ International Symposium on Shock Waves, July 11-16, 2004, Beijing, China

${ }^{8}$ Litchford, R.J., "Development of a Gas-Fed Pulse Detonation Research Engine,” AIAA Paper 2001-3814, 2001.

${ }^{9}$ Stanley, S.B., Stuessy, W.S. and Wilson, D.R., "Experimental Investigation of Pulse Detonation Wave Phenomenon," AIAA Paper 95-2197, 1995.

${ }^{10}$ Gordon S. and McBride, B.J. "Computer Program for Calculation of complex Chemical Equilibrium Compositions and Applications I. Analysis," NASA RP-1311, 1976 (http: //WwW.grc.nasa.gov/WWW/CEAWeb/xWhatCE A. htm) 